\title{
Initial clinical experience with the first drug (sacubitril/valsartan) in a new class - angiotensin receptor neprilysin inhibitors in patients with heart failure with reduced left ventricular ejection fraction in Poland
}

\author{
Marta Kałużna-Oleksy, ${ }^{1,2}$, Jolanta Kolasa ${ }^{3}$, Jacek Migaj ${ }^{1,2}$, Agnieszka Pawlak ${ }^{4,5}$, Małgorzata Lelonek ${ }^{6}$, \\ Jadwiga Nessler ${ }^{7,8}$, Ewa Straburzyńska-Migaj ${ }^{1,2}$ \\ ${ }^{1} 1^{\text {st }}$ Department of Cardiology, University of Medical Sciences, Poznan, Poland \\ ${ }^{2}$ University Hospital of Lord's Transfiguration, Poznan, Poland \\ ${ }^{3}$ Novartis Pharmaceuticals, Medical Department, Warsaw, Poland \\ ${ }^{4}$ Mossakowski Medical Research Centre, Warsaw, Poland \\ ${ }^{5}$ Central Clinical Hospital of the Ministry of Interior, Warsaw, Poland \\ ${ }^{6}$ Department of Noninvasive Cardiology, WAM-CSW Memorial Hospital, Medical University, Lodz, Poland \\ 'Department of Coronary Disease and Heart Failure, Institute of Cardiology, Jagiellonian University, Medical College, Krakow, Poland \\ ${ }^{8}$ John Paul II Hospital, Krakow, Poland
}

\begin{abstract}
Background: Sacubitril/valsartan is the first drug from a new class of angiotensin receptor neprilysin inhibitors (ARNIs) recommended in the new European Society of Cardiology guidelines instead of angiotensin converting enzyme inhibitors (ACEI), or angiotensin receptor blockers (ARB) that are used if ACEI are not tolerated. Sacubitril/valsartan is recommended for further reduction in the risk of hospitalisation or death in outpatients with heart failure with reduced ejection fraction (HFrEF) if symptoms continue despite optimal treatment with ACEI/ARB, beta-blockers, and mineralocorticoid antagonists.

Aim: The aim of this study is to present the initial experience with regard to the effectiveness, tolerance, and safety of sacubitril/valsartan in the outpatient cardiology practice in Poland.

Methods: The study is a retrospective analysis of data obtained through a questionnaire filled in by the physicians who initiated the sacubitril/valsartan treatment in patients with HFrEF between 1 June 2016 and 30 September 2016. Patients were followed-up for three months.

Results: The analysis included data on 28 patients aged $61 \pm 16$ years, of whom $85.7 \%$ were males. The drug was used in patients in New York Heart Association (NYHA) class I-III. In 25 (89.2\%) patients sacubitril/valsartan was started at the lowest dose (24/26 mg BID). During follow-up the sacubitril/valsartan-treated patients had a reduction in HF symptoms assessed using the NYHA functional class $(p=0.001$ ), a significant drop in N-terminal-pro B-type natriuretic peptide levels (mean, from 2900 to $2270 \mathrm{pg} / \mathrm{mL} ; \mathrm{p}=0.008$ ), and improved exercise tolerance, which occurred shortly after treatment initiation — after a mean of 28 days.

Conclusions: It was demonstrated that the use of sacubitril/valsartan in outpatients with HFrEF is safe and is associated with a significant clinical improvement.
\end{abstract}

Key words: ARNI, sacubitril/valsartan, heart failure with reduced ejection fraction

Kardiol Pol 2018; 76, 2: 381-387

Address for correspondence:

Marta Kałużna-Oleksy, MD, PhD, $1^{\text {st }}$ Department of Cardiology, University of Medical Sciences, ul. Długa 1/2, 61-848 Poznań, Poland, e-mail: marta.kaluzna@wp.pl Received: 04.08.2017 Accepted: 26.10.2017 Available as AoP: 24.11.2017

Kardiologia Polska Copyright (C) Polskie Towarzystwo Kardiologiczne 2018 


\section{INTRODUCTION}

Sacubitril/valsartan (molecule code LCZ696) is the first agent in a new class of drugs called angiotensin receptor neprilysin inhibitors (ARNI). The PARADIGM-HF study, which included a population of patients with heart failure with reduced left ventricular ejection fraction (HFrEF), demonstrated superiority of sacubitril/valsartan (ARNI) over enalapril (angiotensin-converting enzyme inhibitor [ACEI]) in the primary endpoint, i.e. cardiovascular death or first hospitalisation for heart failure (HF) [1]. The study showed $20 \%$ reduction in the risk of such events, $20 \%$ reduction in the risk of cardiovascular death, $21 \%$ reduction in the risk of hospitalisation for HF, and $16 \%$ reduction in the risk of all-cause mortality in the LCZ696 group as compared with the enalapril group [1]. It is worth mentioning that PARADIGM-HF was the first study concerning this indication, in which the new molecule was compared with an active drug, but not placebo, as was the case before. Sacubitril/valsartan is generally well-tolerated, with the most common adverse effects in both groups being hypotension, renal dysfunction, hyperkalaemia, and cough. Although symptomatic hypotension occurred significantly more often in the sacubitril/valsartan group as compared with the enalapril group $(2.7 \%$ vs. $1.4 \% ; p<0.001)$, there were no significant differences between the groups in the number of subjects who discontinued treatment for that reason [1]. In the sacubitril/valsartan group, deterioration of renal function, hyperkalaemia, and cough were significantly less frequent than in the enalapril group.

Sacubitril/valsartan was approved for use in European Union countries in November 2015, and in May 2016, i.e. six months after approval, it was included in the European Society of Cardiology (ESC) guidelines on the HFrEF management [2]. Sacubitril/valsartan is therefore recommended as a replacement for ACEI in ambulatory HFrEF patients who remain symptomatic despite optimal therapy and who fit PARADIGM-HF criteria for further reduction in the risk of hospitalisation or death (recommendation class IB) [2]. In the same guidelines, the drug is recommended for use in ventricular arrhythmia (recommendation class IA) in addition to beta-blockers and mineralocorticoid antagonists (MRA). It also has a class I recommendation by the American College of Cardiology/American Heart Association (ACC/AHA) [3]

At present, sacubitril/valsartan is reimbursed in most European countries. In Poland, the reimbursement process is underway, which aims at providing this new therapy to patients with chronic HF with reduced left ventricular ejection fraction (LVEF). Due to limited access to the therapy, clinical experience with the new drug is not as yet considerable. In Poland, sacubitril/valsartan, available under the trade name of Entresto, was approved on 1 June 2016, adding to the range of drugs that improve prognosis and reduce morbidity among HFrEF patients. As experience with using the new drug in clinical practice is limited, data on the initial Polish experience with sacubitril/valsartan need to be collected, systematised, and presented in a pooled analysis.

The aim of this study is to present the initial experience concerning the effectiveness, tolerance, and safety of sacubitril/valsartan in the outpatient cardiology practice in Poland.

\section{METHODS}

The study group comprised patients with HFrEF, who were initiated with sacubitril/valsartan treatment between 1 June 2016 and 30 September 2016, and who were followed-up for three months. The study is a retrospective analysis of data obtained through a questionnaire filled in by the physicians who initiated the drug in the specified period and consented to participate in the study. The questionnaires were commissioned by Novartis and were available for the purposes of this analysis. Special attention was paid to the characteristics of patients in whom sacubitril/valsartan was started in the initial period of its introduction into the Polish market, and to its comparison with the PARADIGM-HF study population, as well as to the degree to which treatment-initiating physicians followed the latest ESC guidelines on HF management published in May 2016 [2] when they were selecting patients to receive sacubitril/valsartan. The paper includes an analysis of the sacubitril/valsartan safety and tolerability profile. Also, the reasons why treatment optimisation was not achieved within three months of initiation and why the drug was discontinued are analysed in this paper.

\section{Statistical analysis}

The statistical analysis was performed using Statistica 12, STATSOFT. The data were presented as absolute numbers with percentages in the case of nominal data and means with standard deviation in the case of continuous data. For the comparison of changes within the study group during subsequent visits, the Wilcoxon test for paired samples was used. A p-value $<0.05$ was considered statistically significant.

\section{RESULTS}

The analysis included data on 28 patients aged $61 \pm 16$ years, of whom $85.7 \%$ were males. The drug was used in patients in New York Heart Association (NYHA) class I-III. LVEF was $15-40 \%$ (mean 25\%). The baseline characteristics are presented in Table 1 . In the majority of patients, non-ischaemic cardiomyopathy was the underlying cause of $\mathrm{HF}$ (18 patients, $64.3 \%$ ). Mean systolic blood pressure (SBP) was $116 \pm 14 \mathrm{mmHg}$, while mean diastolic blood pressure (DBP) was $74 \pm 10 \mathrm{mmHg}$. The mean level of $\mathrm{N}$-terminal-pro B-type natriuretic peptide (NT-proBNP) was $2900 \mathrm{pg} / \mathrm{mL}$ (available for 22 patients only). Cardiac resynchronisation therapy defibrillator (CRT-D) was used in six (21\%) patients and implantable cardioverter-defibrillator (ICD) in 13 (46\%) patients.

Prior to initiation of sacubitril/valsartan, the patients received either ACEI or ARB. ACEI (ramipril, perindopril, 
Table 1. General characteristics of patients started on sacubitril/valsartan

\begin{tabular}{|c|c|c|}
\hline & Mean \pm SD & Range (min-max) \\
\hline Age [years] & $61 \pm 16$ & $26-86$ \\
\hline Sex (male/female) & 24/4 (86/14\%) & \\
\hline NYHA class (mean): & $2.8 \pm 0.5$ & $1-3$ \\
\hline । & $1(3 \%)$ & \\
\hline$\|$ & $5(18 \%)$ & \\
\hline III & $22(79 \%)$ & \\
\hline LVEF [\%] & $25 \pm 8$ & $15-40$ \\
\hline \multicolumn{3}{|l|}{ HF aetiology: } \\
\hline Ischaemic & $18(64 \%)$ & \\
\hline Non-ischaemic & $10(36 \%)$ & \\
\hline Sinus rhythm & $22(79 \%)$ & \\
\hline \multicolumn{3}{|l|}{ Pharmacotherapy: } \\
\hline Beta-blockers & 27 (96\%) & \\
\hline ACEI & $19(68 \%)$ & \\
\hline ARB & $6(21 \%)$ & \\
\hline MRA & $28(100 \%)$ & \\
\hline Systolic BP [mmHg] & $116 \pm 14$ & $95-145$ \\
\hline Diastolic BP [mmHg] & $74 \pm 10$ & $55-93$ \\
\hline Heart rate [bpm] & $72 \pm 12$ & 50-100 \\
\hline Creatinine $[\mu \mathrm{mol} / \mathrm{L}]$ & $107 \pm 27$ & $57-156$ \\
\hline Potassium [mmol/L] & $4.33 \pm 0.35$ & $3.65-5.02$ \\
\hline$I C D$ & $13(46 \%)$ & \\
\hline CRT-D & $6(21 \%)$ & \\
\hline No-device & $9(33 \%)$ & \\
\hline
\end{tabular}

Data are presented as mean \pm standard deviation (SD) or number (percentage). ACEI - angiotensin converting enzyme inhibitors; ARB — angiotensin receptor blockers; BP — blood pressure; CRT-D — cardiac resynchronisation therapy defibrillator; HF - heart failure; ICD — implantable cardioverter-defibrillator; LVEF — left ventricular ejection fraction; NYHA — New York Heart Association; MRA — mineralocorticoid antagonists lisinopril, or zofenopril) were used in 19 (67.9\%) patients while ARB (valsartan or losartan) was in six (21.4\%) patients (Fig. 1). Twenty-eight (100\%) patients received an MRA while 27 (96\%) received a beta-blocker. The mean daily dose of $A C E I / A R B$ was equivalent to $10 \mathrm{mg}$ enalapril. Detailed data on pharmacological treatment before the initiation of the sacubitril/valsartan treatment is presented in Table 2. Sacubitril/valsartan is available in three doses, the target dose being 97/103 mg twice daily (BID). The initial dose recommended in the Summary of Product Characteristics [4] (sacubitril/valsartan 49/51 mg BID) was used only in three patients, and two of them required dose reduction due to symptomatic hypotension. In 25 (89.2\%) patients sacubitril/valsartan was started at the lowest dose (24/26 mg BID). At consecutive visits, the dose was increased to $49 / 51 \mathrm{mg}$ BID in 11 patients. Finally, in the follow-up period, the dose was increased to the target dose of 97/103 mg BID only in one patient, while one patient received 97/103 mg in the morning and $49 / 51 \mathrm{mg}$ in the evening. Only one patient discontinued from the new drug after the first follow-up visit, after four months of treatment, because of its high price.

In all the patients who were included in the analysis, sacubitril/valsartan was started in the outpatient setting. In the three-month follow-up period, the first follow-up visit was held after a mean of 28 days (7-89 days). Twelve (42\%) patients came to two follow-up visits and eight (28\%) came to three follow-up visits during the follow-up period. The second visit was held after a mean of 50 days from treatment initiation (33-100 days), while the third visit was held after a mean of 70 days (63-89 days). The visits were related to the sacubitril/valsartan dose adjustment as well as assessment of its tolerance and safety.

During follow-up the sacubitril/valsartan-treated patients had a statistically significant reduction in HF symptoms assessed using the NYHA functional class, with a mean drop from 2.8 to 2.1 ( $p=0.001)$, and a significant decrease in the mean NT-proBNP levels from 2900 to $2270 \mathrm{pg} / \mathrm{mL}$

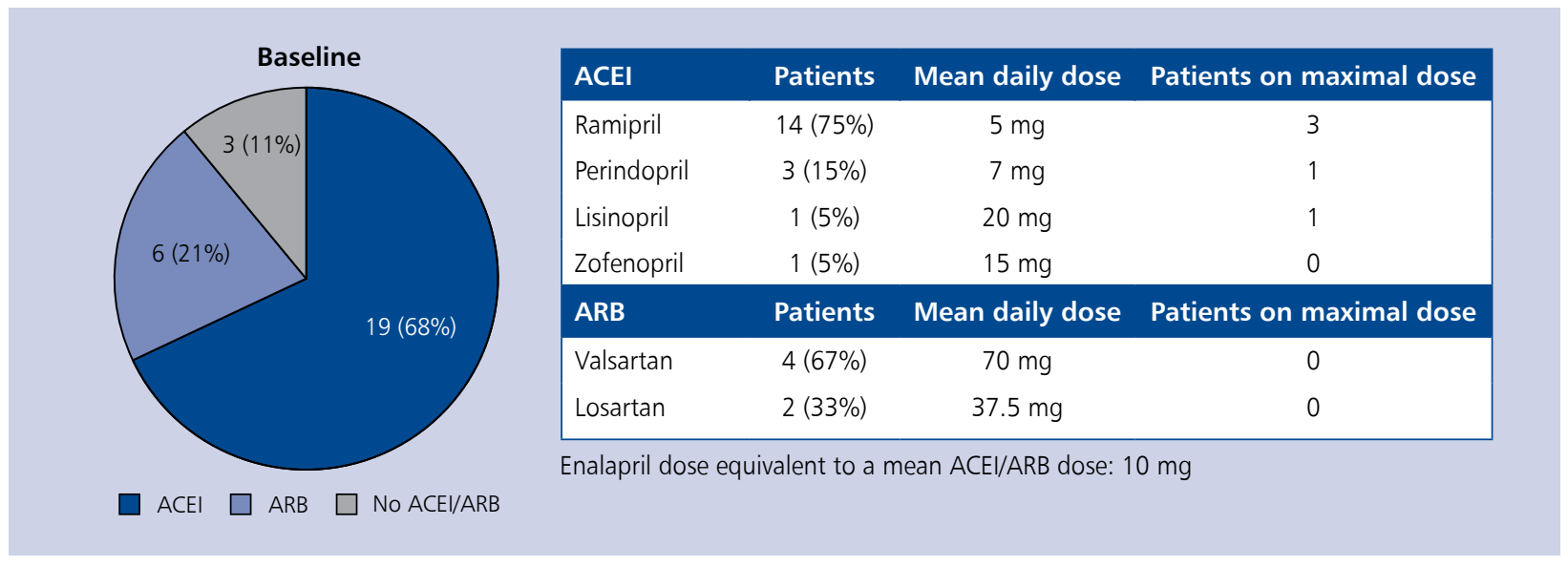

Figure 1. Initial treatment - angiotensin converting enzyme inhibitors/angiotensin receptor blockers (ACEI/ARB) 
Table 2. Pharmacological treatment before the sacubitril/ /valsartan initiation

\begin{tabular}{|c|c|c|}
\hline & $\begin{array}{l}\text { No. of patients } \\
\text { (proportion) }\end{array}$ & $\begin{array}{c}\text { Mean daily dose } \\
{[\mathrm{mg}]}\end{array}$ \\
\hline \multicolumn{3}{|l|}{ Loop diuretics } \\
\hline Furosemide & $16(57 \%)$ & 137.5 \\
\hline Torasemide & $11(39 \%)$ & 26.8 \\
\hline \multicolumn{3}{|l|}{ Beta-blockers } \\
\hline Carvedilol & $11(39 \%)$ & 26 \\
\hline Bisoprolol & $8(29 \%)$ & 4.5 \\
\hline Metoprolol & $5(18 \%)$ & 120 \\
\hline Nebivolol & $3(11 \%)$ & 2.5 \\
\hline \multicolumn{3}{|l|}{ ACEI } \\
\hline Ramipril & $14(50 \%)$ & 4.9 \\
\hline Perindopril & $3(11 \%)$ & 7.5 \\
\hline Lisinopril & $1(4 \%)$ & 20 \\
\hline Zofenopril & $1(4 \%)$ & 15 \\
\hline \multicolumn{3}{|l|}{ ARB } \\
\hline Valsartan & $4(14 \%)$ & 70 \\
\hline Losartan & $2(7 \%)$ & 37.5 \\
\hline \multicolumn{3}{|l|}{ MRA } \\
\hline Spironolactone & $6(21 \%)$ & 29.2 \\
\hline Eplerenone & $22(79 \%)$ & 36.4 \\
\hline Ivabradine & $5(18 \%)$ & 10 \\
\hline Digoxin & $4(14 \%)$ & 0.175 \\
\hline Amiodarone & $4(14 \%)$ & 200 \\
\hline
\end{tabular}

Data are presented as number (percentage). ACEI — angiotensin converting enzyme inhibitors; $A R B$ - angiotensin receptor blockers; MRA — mineralocorticoid antagonists

$(p=0.008)$. Patients reported a considerable improvement in their well-being and increased physical activity. Also, slight decreases in mean SBP (from 116 to $110 \mathrm{mmHg}$ ), mean DBP (from 74 to $70 \mathrm{mmHg}$ ), and heart rate (from 72 to $70 \mathrm{bpm}$ ) were observed (Table 3). On the other hand, insignificant trends toward increases in mean creatinine levels (from
107 to $114 \mu \mathrm{mol} / \mathrm{L}$ ) and serum potassium levels (from 4.33 to $4.63 \mathrm{mmol} / \mathrm{L}$ ) were observed.

There were no significant changes in HF treatment observed during the follow-up period. Because of hypotonia, in one patient alpha-blocker was discontinued, in a second patient a long-acting nitrate was discontinued, and in a single patient, the dose of furosemide was reduced by half. The change in NYHA class analysis was performed in relation to the baseline treatment. The most marked improvement in NYHA functional class was noted in six patients who had been treated with ARB (mean improvement from 2.8 to 1.8).

\section{Safety and adverse events}

Of 28 patients with HFrEF, sacubitril/valsartan was continued during the follow-up period in 26 patients. Drug discontinuations were caused by high price for one patient and due to symptomatic hypotension in another patient. Only in one patient was the dose increased to $97 / 103 \mathrm{mg}$ BID, while one patient received 97/103 $\mathrm{mg}$ in the morning and 49/51 $\mathrm{mg}$ in the evening. Of the patients in whom treatment was started with 49/51 mg BID, two required dose reduction by half due to symptomatic hypotension. Adverse events developed in eight patients: skin symptoms in one and hypotension in seven (Tables 4 and 5). The use of sacubitril/valsartan in the analysed group was safe and was not associated with significant adverse events. During the follow-up period, the reduction in both SBP and DBP (by $6 \mathrm{mmHg}$ and $4 \mathrm{mmHg}$, respectively) was not significant. No patient was hospitalised or died.

\section{DISCUSSION}

Despite the strong evidence for improved prognosis in patients with heart failure with reduced ejection fraction as well as high ESC recommendation class (IB), sacubitril/valsartan is rarely used in Poland. As the analysis of the initial experience has demonstrated, patients achieve rapid and significant clinical improvement as expressed by reduced NYHA class and drops in the NT-proBNP levels. In addition, treatment is safe and well-tolerated. Only one patient required drug discontinuation due to adverse reaction (hypotension). No patient was hospitalised during the follow-up period.

Table 3. Changes in chosen parameters between the treatment initiation and the first follow-up visit after a mean of 28 days

\begin{tabular}{lccc|} 
& Baseline value & First follow-up visit value & p \\
\hline NYHA class & $2.8 \pm 0.5$ & $2.1 \pm 0.7$ & 0.001 \\
Systolic BP $[\mathrm{mmHg}]$ & $116 \pm 14$ & $110 \pm 11$ & 0.009 \\
Diastolic BP $[\mathrm{mmHg}]$ & $74 \pm 10$ & $70 \pm 6$ & 0.008 \\
Heart rate $[\mathrm{bpm}]$ & $72 \pm 12$ & $70 \pm 7$ & NS \\
NT-proBNP $[\mathrm{pg} / \mathrm{mL}]$ & $2900 \pm 1874$ & $2270 \pm 1682$ & 0.008 \\
Creatinine $[\mu \mathrm{mol} / \mathrm{L}]$ & $107 \pm 27$ & $114 \pm 25$ & NS \\
Potassium $[\mathrm{mmol} / \mathrm{L}]$ & $4.33 \pm 0.3$ & $4.63 \pm 0.4$ & NS \\
\hline
\end{tabular}

Data are presented as mean \pm standard deviation. BP — blood pressure; NT-proBNP - N-terminal-pro B-type natriuretic peptide; NYHA — New York Heart Association 
Table 4. Changes in sacubitril/valsartan dosage during the three-month follow-up

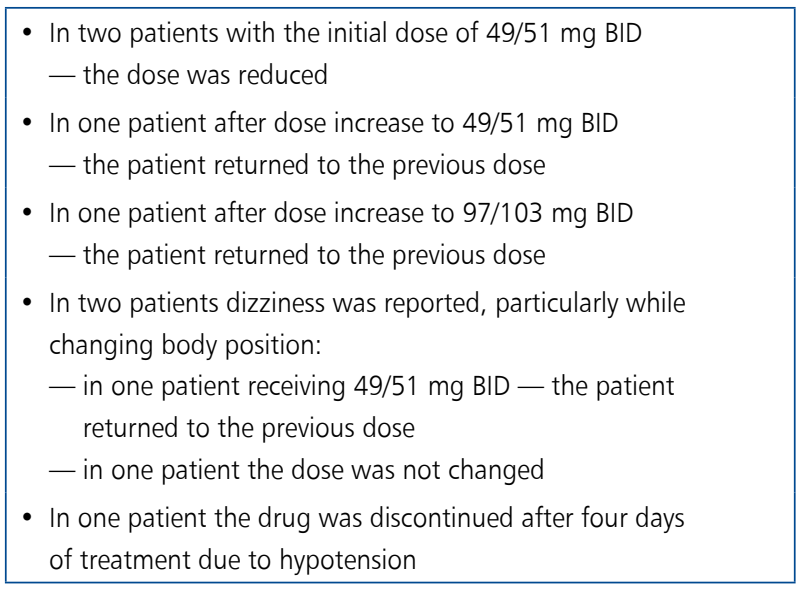

BID - twice daily

Table 5. Adverse events

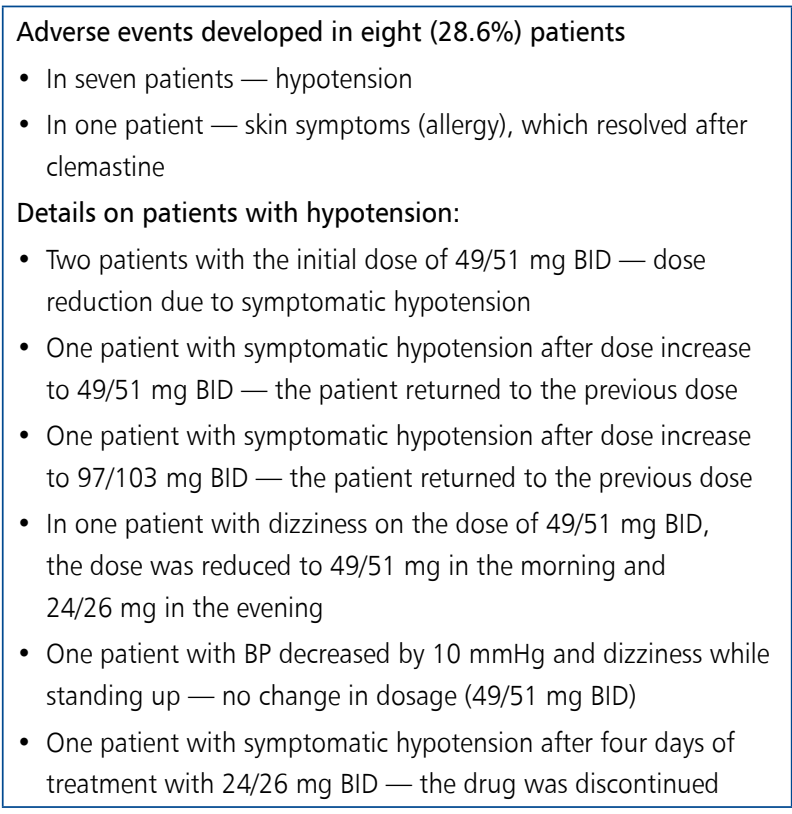

BID — twice daily

Sacubitril/valsartan (molecule code LCZ696) offers a new hope to patients with HFrEF. The drug secured a high position in the 2016 guidelines on heart failure of the ESC and the ACC/AHA [2, 3]. The paper on the initial experience with sacubitril/valsartan in Polish patients with HFrEF provides valuable information on the actual management of $\mathrm{HF}$ and on what is important when the drug is initiated and continued in the outpatient setting.

In all the patients analysed, sacubitril/valsartan was initiated in the outpatient setting, in accordance with recommendations issued by various societies and associations on cardiac diseases, $36 \mathrm{~h}$ after ACEI discontinuation. The follow-up data demonstrated the safety of such therapy.

Starting sacubitril/valsartan in the outpatient setting, as current experience shows, should be associated with necessary patient monitoring during frequent follow-up visits. Optimally, the first follow-up visit should be held about two weeks after drug initiation, while the next one - about six weeks after drug initiation (i.e. four weeks after the first visit). It is important in light of the possible need for sacubitril/valsartan dose adjustment as well as safety monitoring. The decision on further dose optimisation should be preceded by the assessment of renal function and potassium levels. The patient should be informed about the risk of hypotension and the need for daily blood pressure measurements in the home setting. To this purpose, the HF Patient Passport available from the Working Group on Heart Failure of the Polish Cardiac Society may be used.

According to the sacubitril/valsartan Summary of Product Characteristics, treatment should be initiated with the dose of 49/51 mg BID in patients who well tolerated high doses of $\mathrm{ACEI} / \mathrm{ARB}$, with normal renal and hepatic function and SBP above $110 \mathrm{mmHg}$, as in the PARADIGM-HF study [4]. Most Polish physicians, however, started with lower doses. Treatment was started with the recommended dose of 49/51 mg BID only in three of 28 patients, even though five patients had received high doses of $\mathrm{ACEl}$ and one - ARB. In two of the three patients, the dose was reduced due to hypotension. We do not know whether or not another attempt at dose increase was made. It is worth noting that if the patient has a poorer drug tolerance (i.e. SBP below $95 \mathrm{mmHg}$, signs of hypotension, hyperkalaemia, or renal dysfunction), dose adjustment or discontinuation of other drugs (such as diuretics, nitrates, and calcium channel blockers) that have lower impact on prognosis, should be considered in order to return to a higher dose of sacubitril/valsartan. The TITRATION study demonstrated that both the conservative strategy (initially 24/26 mg BID over two weeks, and subsequently 49/51 mg BID over three weeks up-titrated to the target dose of 97/103 mg BID) and condensed strategy (initially 49/51 mg BID over two weeks up-titrated to the target dose of $97 / 103 \mathrm{mg}$ BID) enables achievement of the target dose in a similarly high percentage of patients (conservative strategy, 84.3\% vs. condensed strategy, $77.8 \% ; p=0.078$ ) [5]. The conservative dosage strategy was effective especially in patients who had received low doses of ACEI/ARB or had not received such therapy, and the target dose was achieved in $84.9 \%$ of patients vs. $73.6 \%$ in the condensed strategy group. This difference was statistically significant $(p=0.03)$. It is worth noting that randomisation to the two dosage regimens was preceded by a five-day run-in period when the 24/26 mg (i.e. the lowest available) dose was administered in an open-label fashion to all patients [5].

In the present group, initiation of treatment with the lowest doses was most likely related to the lack of experience, 
which prompted greater caution in using a new drug class representative. Such an approach in the initial period, when experience with the new drug is being gained, was also suggested by experts from the Working Group on Heart Failure of the Polish Cardiac Society [6]. Once good tolerance has been confirmed, the drug dose should be up-titrated to the maximum dose of 97/103 mg BID. As the analysis shows, in the case of patients starting the new therapy, follow-up visits were irregular — only eight (29\%) patients came to three visits in the three-month follow-up period, which could hinder treatment intensification and outcome control. Nevertheless, one should bear in mind that aiming at optimum doses of sacubitril/valsartan and other prognosis-affecting drugs should constitute an essential element of the outpatient care.

The PARADIGM-HF study demonstrated superiority of sacubitril/valsartan over enalapril at every dose, even at low doses [7]. A low dose of sacubitril/valsartan is particularly useful in initiating it in patients who have not received ACEI/ARB or received low doses of these drugs [5], as well as individuals with renal or hepatic dysfunction or low baseline SBP ranging from 100 to $110 \mathrm{mmHg}$. The American guidelines recommend this more liberal approach to sacubitril/valsartan treatment [3]. They do not provide for the need for ACEI dose optimisation prior to initiating, switching to, or de novo initiating sacubitril/valsartan [3].

The analysis showed that not all patients had their NT-proBNP levels tested when new treatment eligibility was assessed although, according to the ESC guidelines, treatment should be initiated in patients with a profile similar to that in the PARADIGM-HF study, in which the NT-proBNP level was one of the inclusion criteria [2]. In Poland, such a test is practically unavailable in the outpatient setting; therefore, using the NT-proBNP level as an eligibility criterion is very difficult.

Even though in most patients only the initial sacubitril/valsartan dose of 24/26 mg was used, a significant improvement in the patient well-being was achieved. Patients reported increased physical activity and improved exercise tolerance. No patient required hospitalisation or died during the follow-up period, despite 22 (79\%) patients having been NYHA class III when sacubitril/valsartan was initiated and having been hospitalised for decompensated HF on a number of occasions.

Undoubtedly, the current cost of sacubitril/valsartan treatment in Poland is high and may constitute a barrier to its initiation. An analysis of the profile of patients selected to the initial experience with the new drug shows that physicians, probably because of its high cost, decide to use sacubitril/valsartan in patients with advanced HF, who fail to achieve satisfactory improvement with standard therapy. It is worth emphasising that in PARADIGM-HF 70\% of patients were in NYHA class II and most of them had not been hospitalised for decompensated $\mathrm{HF}$, which means that the drug should be used in a considerably wider population of HFrEF patients.
Initiation of sacubitril/valsartan in an outpatient setting is safe and brings about satisfactory effects as soon as within the first weeks, which are reported by patients as improved clinical status reflected by improved NYHA class or exercise tolerance. Nevertheless, follow-up visits are necessary to ensure adequate dose optimisation and control. At present, the high cost of sacubitril/valsartan appears to be the major obstacle to its wide use in Poland. Given that sacubitril/valsartan is the first pharmacotherapy that has been approved in the last 10 years for the treatment of symptomatic chronic HFrEF, which reduces morbidity and mortality, it is of key importance to provide access to sacubitril/valsartan to a broad population of patients who require pharmacotherapy optimisation according to the current guidelines.

\section{Limitations of the study}

It was a retrospective study. The size of the analysed group was limited, and patients were not treated according to the established protocol. Further follow-up and clinical experience are needed.

\section{CONCLUSIONS}

The use of sacubitril/valsartan in outpatients with HFrEF is safe and is associated with a significant clinical improvement, as reflected by reduced NYHA class and NT-proBNP level. What is important, a noticeable improvement in exercise tolerance was achieved soon after treatment initiation - after a mean of 28 days.

\section{Acknowledgements}

We wish to thank the following persons for the preparation of the questionnaires: A. Gziut (Warsaw), S. Jasek (Warsaw), P. Kałmucki (Poznan), M. Kłączyński (Poznan), M. Kochmański (Warsaw), A. Mamzer-Dmochowska (Lodz), A. Mirek (Warsaw), P. Rubiś (Krakow), M. Starczewska (Warsaw), W. Szychta (Warsaw), L. Urbańska (Poznan).

Conflict of interest: M. Kałużna-Oleksy: participation in clinical trials Novartis; J. Kolasa: employee of Novartis Pharmaceuticals, J. Migaj: participation in clinical trials Novartis; A. Pawlak: fees for lectures and consultations Novartis, M. Lelonek: participation in clinical trials Novartis, fees for lectures and consultations Novartis, J. Nessler: participation in clinical trials Novartis, fees for lectures and consultations Novartis; Ewa Straburzyńska-Migaj: participation in clinical trials Novartis, fees for lectures and consultations Novartis.

\section{References}

1. McMurray J, Packer M, Desai A, et al. Angiotensin-Neprilysin Inhibition versus Enalapril in Heart Failure. New Engl J Med. 2014; 371(11): 993-1004, doi: 10.1056/nejmoa1409077.

2. Ponikowski P, Voors AA, Anker SD, et al. [2016 ESC Guidelines for the diagnosis and treatment of acute and chronic heart failure]. Kardiol Pol. 2016; 74(10): 1037-1147, doi: 10.5603/KP.2016.0141, indexed in Pubmed: 27748494. 
3. Yancy CW, Jessup M, Bozkurt B, et al. 2016 ACC/AHA/HFSA Focused Update on New Pharmacological Therapy for Heart Failure: An Update of the 2013 ACCF/AHA Guideline for the Management of Heart Failure: A Report of the American College of Cardiology/American Heart Association Task Force on Clinical Practice Guidelines and the Heart Failure Society of America. J Am Coll Cardiol. 2016; 68(13): 1476-1488, doi: 10.1016/j. jacc.2016.05.011, indexed in Pubmed: 27216111.

4. Entresto, INN-sacubitril/valstartan. SUMMARY OF PRODUCT CHARACTERISTICS. 2015.

5. Senni M, McMurray JJV, Wachter R, et al. Initiating sacubitril/valsartan (LCZ696) in heart failure: results of TITRATION, a double-blind, randomized comparison of two uptitration regimens. Eur J Heart Fail. 2016; 18(9): 1193-1202, doi: 10.1002/ejhf.548, indexed in Pubmed: 27170530.
6. Straburzyńska-Migaj E, Nessler J, Gruchała M, et al. Sacu-bitril/valsartan for treatment of chronic heart failure with reduced ejection fraction. Can all patients benefit? A position statement paper of experts of the Heart Failure Working Group of the Polish Cardiac Society. Kardiol Pol. 2017; 75(3): 286-293, doi: 10.5603/KP.2017.0056, indexed in Pubmed: 28326531.

7. Vardeny O, Claggett B, Packer M, et al. Prospective Comparison of ARNI with ACEI to Determine Impact on Global Mortality and Morbidity in Heart Failure (PARADIGM-HF) Investigators. Efficacy of sacubitril/valsartan vs. enalapril at lower than target doses in heart failure with reduced ejection fraction: the PARADIGM-HF trial. Eur J Heart Fail. 2016; 18(10): 1228-1234, doi: 10.1002/ejhf.580, indexed in Pubmed: 27283779 .

Cite this article as: Kałużna-Oleksy M, Kolasa J, Migaj J, et al. Initial clinical experience with the first drug (sacubitril/valsartan) in a new class - angiotensin receptor neprilysin inhibitors in patients with heart failure with reduced left ventricular ejection fraction in Poland. Kardiol Pol. 2018; 76(2): 381-387, doi: 10.5603/KP.a0230. 